Results: Of the 1,224 patients fulfilling the inclusion criteria, $93(7.6 \%)$ switched therapy and 1,131 (92.4\%) did not switch therampy after not achieving an adequate response on the initial b/tsDMARD. At BL, $42.5 \%$ and $70.0 \%$ of patients had no meaningful improvement to their prior therapy based on $\geq 6$ and $\geq 12$-unit change, respectively; mean (SD) age was 53.1 (14.0) years; duration of RA 10.7 (10.4) years; CDAI 22.2 (10.8); 81.7\% were female; $64.5 \%$ had MDA, 35.5\% had HDA; $21.5 \%$ reported being disabled, $24.7 \%$ were current smokers, and $50 \%$ were obese. In terms of prior biologic use $57.0 \%, 22.6 \%$, and $20.4 \%$ had been on 1,2 , and $3+$, respectively. From $B L$ to $F / U$, meaningful worsening occurred in $30.1 \%$ and $12.9 \%$ using a threshold of 6 and 12 , respectively, with the remaining patients experiencing meaningful improvement or no meaningful change (Figure 1).

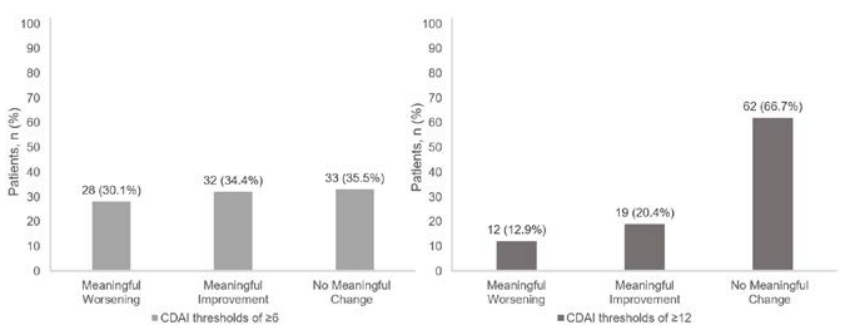

Figure 1. Meaningful Worsening, Meaningful Improvement, and No Meaningful Change Based on CDAl Change Thresholds of $\geq 6$ and $\geq 12$ From $B L$ to $F / U(N=93)$

Conclusion: In our analysis, a large proportion of patients who initiated a biologic/JAKi and experienced some improvement but failed to attain LDA or remission, did not switch therapy within approximately a year. This analysis consisted of many patients who did not have a meaningful response to their prior biologic/ JAKi, patients who had received multiple prior biologics, and a large portion of patients with poor prognostic factors. Despite this, the proportion of patients with meaningful worsening was low compared with most patients who had either meaningful improvement or no meaningful change. Additional research is warranted to understand the reasons for not switching and whether the likelihood of a meaningful change correlates with prior response, poor prognosis, or other factors.

Acknowledgements: Amy Praestgaard (Sanofi) contributed to the statistical analysis for this abstract. Medical writing support for this abstract was provided by Krishna Kammari (Sanofi).

Disclosure of Interests: Jeffrey Curtis Grant/research support from: and personal fees from AbbVie, Amgen, BMS, CORRONA, Eli Lily, Janssen, Myriad, Pfizer, Roche, Regeneron, Radius, UCB, outside the submitted work, Stefano Fiore Shareholder of: Sanofi, Employee of: Sanofi. In addition, he has a patent EP 19306553.9; USPTO \#s 62/799,698; 62/851,474; 62/935,395 issued, Kerri Ford Shareholder of: Sanofi, Employee of: Sanofi, Judson Janak: None declared, Hong Chang: None declared, Dimitrios A Pappas Employee of: CORRONA LLC. He has previously acted as a consultant for Sanofi, Abbvie, Gtech Roche Hellas, and Novartis. He has an equity interest in CORRONA LLC. and is on the Board of directors of the CORRONA research foundation, Taylor Blachley: None declared, Kelechi Emeanuru: None declared, Vivian Bykerk Grant/research support from: reports grants from Amgen, BMS, UCB, and Novartis were given to institution, that grants from the $\mathrm{NIH}, \mathrm{PCORI}$, and $\mathrm{CIHR}$ were given to institutions which whom she is affiliated, and that she has received personal fees from Amgen, Gilead, BMS, Pfizer, Sanofi Aventis, Roche, UCB and Regeneron, outside the submitted work.

DOI: 10.1136/annrheumdis-2021-eular.544

\section{POS0595 ABATACEPT IN USUAL AND IN NON-SPECIFIC INTERSTITIAL PNEUMONIA ASSOCIATED TO RHEUMATOID ARTHRITIS. NATIONAL MULTICENTER STUDY OF 190 PATIENTS}

B. Atienza-Mateo ${ }^{1}$, C. Fernández-Díaz ${ }^{2}$, S. Castañeda ${ }^{2}$, R. Melero ${ }^{3}$, F. Ortiz Sanjuan $^{4}$, I. Casafont-Solé ${ }^{5}$, S. C. Rodriguez-García ${ }^{6}$, I. Ferraz-Amaro ${ }^{7}$, M. A. González-Gay ${ }^{1}$, R. Blanco ${ }^{1}$ on behalf of Members of the Spanish Collaborative Group of Abatacept in Interstitial Lung Disease Associated with Rheumatoid Arthritis: Clara Aguilera-Cros (H.U. Virgen del Rocío, Sevilla), Ignacio Villa (H. Sierrallana, Torrelavega), Enrique Raya-Alvarez (H.U. San Cecilio, Granada), Clara Ojeda-García (H.U. Virgen Macarena, Sevilla), María G. Bonilla Hernán (H.U. La Paz, Madrid), Ana M. López-Robles (H.U. Vall d'Hebron, Barcelona), Luis Arboleya-Rodríguez (H.U. Central de Asturias, Asturias), Javier Narváez García (H.U. Bellvitge, Barcelona), Evelin C. Cervantes Pérez (H.U. de Santiago, Santiago de Compostela, A Coruña), OlgaMaiz-Alonso (H.U. Donostia, Gipuzkoa), María N. Alvarez-Rivas (H.U. Luca Augusti, Lugo), Iván Cabezas-Rodríguez (H.U. Río Hortega, Valladolid), Eva Salgado-Pérez (C.H.U. Ourense, Ourense), Cristina Hidalgo-Calleja (H.U. de Salamanca, Salamanca), Sabela Fernández-Aguado (H.U. Cabueñes, Asturias), Jesús C. Fernández-López (C.H.U. A Coruña), Alejandro Olivé, Samantha Rodríguez-Muguruza (H.U. GermansTrias i Pujol, Barcelona), Raquel Almodóvar-González (H.U. Fundación Alcorcón, Madrid), Carmen Carrasco-Cubero (C.H.U. Infanta Cristina, Badajoz), Antonio Juan-Mas (H. Son Llàtzer, Palma de Mallorca), Raúl Castellanos-Moreira (H.U. Clinic. Barcelona), Iñigo Hernández Rodríguez (C.H.U. de Vigo, Vigo), Neus Quillis-Marti (H. Vinalopo Elche), José A. Bernal-Vidal (H. Marina Baixa, Villajoyosa), Angel García-Aparicio (H. Virgen de la salud, Toledo), Sonia Castro-Oreiro (H.U. Joan XXIII, Tarragona), Julia Fernández-Melón (H. Son Espases, Palma de Mallorca), Paloma Vela Casasempere(H. U. Alicante, Alicante), María C. Fito, Carmen González-Montagut (C.U. Navarra, Navarra), Manuel RodríguezGómez (C.H.U. Ourense, Ourense), Trinidad Pérez-Sandoval, Miriam RetuertoGuerrero (H.U. León), Deseada Palma-Sánchez (H. Rafael Mendez, Lorca), José L. Andreu (H. U. Puerta del Hierro), Patricia Carreira-Delgado (H. 12 de Octubre, Madrid), Lorena Expósito-Pérez (H.U. de Canarias, Tenerife), Juan RD Jiménez- de Aberásturi, Ana Ruibal-Escribano (H.U. Txagorritxu, Araba), Ana Urruticoechea-Arana (H. Can Misses, Ibiza), Rosa Expósito-Molinero (H. Laredo, Laredo), Rubén López Sánchez (H. Negrín Las Palmas), Manuel J. Moreno-Ramos (H. Virgen de la Arrixaca, Murcia), Isabel Serrano-García (H. Puerta del Mar Cadiz), Blanca García-Magallón (H. San Jorge Huesca), José María Andreu Ubero (H. Virgen de las Nieves Granada), Natalia MenaVázquez (H.R.U Málaga), Iván Castellvi-Barranco (H.U. De la Santa Creu i Sant Pau, Barcelona), Carmen González-Montagut (H. U. Valladolid, Valladolid), Juan Blanco-Madrigal (H. Basurto, Bilbao), Pilar Morales-Garrido (H.U. San Cecilio, Granada), Cilia Peralta-Ginés (H. Lozano Blesa, Zaragoza), Mireia López-Corbeto (H.U. Vall d’Hebron, Barcelona), Sergio Ordóñez-Palau (H. Lleida, Lleida), Andrea García-Valle (H. U Palencia), Susana Romero-Yuste (H.U. Pontevedra, Pontevedra).. ${ }^{1}$ Hospital Universitario Marqués de Valdecilla, IDIVAL, Rheumatology, Santander, Spain; ${ }^{2}$ Hospital Universitario La Princesa, Rheumatology, Madrid, Spain; ${ }^{3}$ Complejo Hospitalario Universitario de Vigo, Rheumatology, Vigo, Spain; ${ }^{4}$ Hospital Universitario La Fe, Rheumatology, Valencia, Spain; ${ }^{5}$ Hospital Universitario Germans Trias i Pujol, Rheumatology, Barcelona, Spain; ${ }^{6}$ Hospital Universitario Clinic, Rheumatology, Barcelona, Spain; ${ }^{7}$ Hospital Universitario de Canarias, Rheumatology, Santa Cruz de Tenerife, Spain

Background: Interstitial lung disease (ILD) is a severe complication of Rheumatoid Arthritis (RA). Usual interstitial pneumonia (UIP) is considered to be more frequent and severe in RA than non-specific interstitial pneumonia (NSIP). Abatacept (ABA) and Rituximab have demonstrated efficacy in RA-ILD [1-3].

Objectives: To compare the efficacy of ABA in RA-ILD patients according to radiological patterns of UIP or NSIP.

Methods: From an observational multicenter study of 263 RA-ILD patients treated with ABA, we selected those with UIP or NSIP [2]. We analyzed in the 2 groups from baseline up to 24 months the following outcomes: a) Forced Vital Capacity (FVC), b) Carbon monoxide diffusing capacity (DLCO), c) Chest High Resolution Computed Tomography (HRCT), and d) dyspnea. Differences between final follow-up and basal visit were calculated as the average difference and $95 \%$ Confidence Interval $(95 \% \mathrm{Cl})$. Multivariable linear regression was used to assess the differences between the 2 groups.

Results: We studied 190 patients with UIP $(n=106)$ and NSIP $(n=84)$. Patients with UIP were older, had more positivity for rheumatoid factor and had received more sulfasalazine (Table 1). ILD duration up to ABA initiation was relatively short in both groups, with a median [IQR] of 16 [4-50] and 11 [2-36] months in UIP and NSIP patterns, respectively. Mean baseline values of FVC and DLCO were > $80 \%$ and $>60 \%$, respectively, in the 2 groups, with a lower FVC in UIP ( $82 \%$ vs $89 \%$ in NSIP, $p<0.05$ ). The evolution of FVC and DLCO is shown in Figure 1. Both parameters remained stable during 24 months of ABA therapy. Available chest HRCT images improved/ stabilized in $73.1 \%$ and $72.9 \%$ of UIP and NSIP patterns, respectively. With multivariable linear regression analysis, no differences were found in the changes of FVC, DLCO, or HRCT images. Stabilization or improvement of dyspnea was found in $91 \%$ and $95 \%$ of UIP and NSIP patterns, respectively.

Conclusion: $A B A$ seems to be equally effective in stabilizing of DLCO, FVC and HRCT in UIP and NSIP in RA-ILD. Our results suggest that an early administration of $A B A$ in ILD, before significant structural lung damage development, may be preferable to prevent interstitial progression, regardless of the radiological pattern.

\section{REFERENCES:}

[1] Fernández-Díaz C, et al. Semin Arthritis Rheum. 2018 Aug;48(1):22-27. doi: 10.1016/j.semarthrit.2017.12.012

[2] Fernández-Díaz C, et al. Rheumatology (Oxford). 2020 Dec 1;59(12):39063916. doi: 10.1093/rheumatology/keaa621

[3] Atienza-Mateo B, et al. J Clin Med. 2020 Sep 23;9(10):3070. doi: 10.3390/ jcm9103070 
Table 1. Main general features at baseline.

\begin{tabular}{llll}
\hline & UIP $(\mathbf{n}=\mathbf{1 0 6})$ & NSIP $(\mathbf{n}=\mathbf{8 4})$ & p value \\
\hline Age, years mean \pm SD & $66 \pm 10$ & $63 \pm 10$ & $\mathbf{0 . 0 4 9}$ \\
Women, $\mathrm{n}(\%)$ & $59(56)$ & $49(58)$ & 0.71 \\
Smoker ever, $\mathrm{n}(\%)$ & $51(48)$ & $45(54)$ & 0.46 \\
ILD duration up toABA, months, median [IQR] & $16[4-50]$ & $11[2-36]$ & 0.57 \\
RF & $100(94)$ & $71(85)$ & $\mathbf{0 . 0 4 1}$ \\
ACPA, $\mathrm{n}(\%)$ & $96(91)$ & $75(89)$ & 0.83 \\
FVC (\% of the predicted), mean \pm SD & $82 \pm 21$ & $89 \pm 19$ & $\mathbf{0 . 0 2 5}$ \\
DLCO (\% of the predicted), mean \pm SD & $63 \pm 19$ & $65 \pm 16$ & 0.46 \\
ABA monotherapy, $\mathrm{n}(\%)$ & $45(42)$ & $41(49)$ & 0.38 \\
ABA combined+ MTX // +other cDMARD, $\mathrm{n}(\%)$ & $15(14) / / 46(43)$ & $16(19) / / 27(32)$ & 0.17 \\
Prednisone at baseline, mg/day, median [IQR] & $7.5[5-10]$ & $10[5-10]$ & 0.20 \\
Previous immunosuppressive therapy, $n(\%)$ & & & \\
MTX & $81(76)$ & $68(81)$ & 0.45 \\
Leflunomide & $48(45)$ & $31(37)$ & 0.25 \\
Sulfasalazine & $16(15)$ & $5(6)$ & $\mathbf{0 . 0 4 6}$ \\
Hydroxychloroquine & $24(23)$ & $16(19)$ & 0.55 \\
Anti-TNF drugs & $37(35)$ & $30(36)$ & 0.80 \\
Rituximab & $19(18)$ & $15(18)$ & 0.99 \\
Tocilizumab & $12(11)$ & $12(14)$ & 0.54 \\
\end{tabular}

ABA, abatacept; ACPA, anti-citrullinated protein antibodies; DMARD, disease-modifying antirheumatic drug, ILD, Interstitial lung disease; MTX, methotrexate; NSIP, non-specific interstitial pneumonia; RA, rheumatoid arthritis; TNF, tumor necrosis factor; UIP, usual interstitial pneumonia.

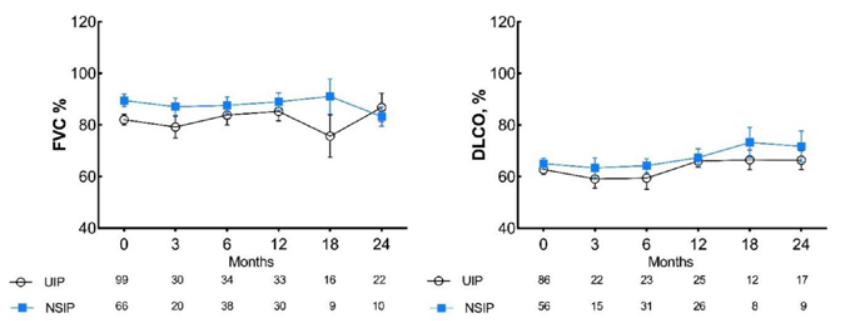

Figure 1. Evolution of pulmonary function tests in RA-ILD patients with UIP and NSIP patterns. FVC and DLCO are expressed as mean $(95 \% \mathrm{Cl})$ and compared between the 2 groups.

Acknowledgements: the Spanish Collaborative Group of Abatacept in Interstitial Lung Disease Associated with Rheumatoid Arthritis

Disclosure of Interests: Belén Atienza-Mateo: None declared, Carlos Fernández-Díaz Speakers bureau: Brystol Myers Squibb, Santos Castañeda: None declared, Rafael Melero: None declared, FRANCISCO ORTIZ SANJUAN: None declared, Ivette Casafont-Solé: None declared, Sebastián C Rodriguez-García: None declared, Iván Ferraz-Amaro: None declared, Miguel A González-Gay: None declared, Ricardo Blanco Speakers bureau: Brystol Myers Squibb DOI: 10.1136/annrheumdis-2021-eular.711

\section{POS0596 THE THERAPEUTIC TARGET IS REACHED MORE OFTEN IN RA PATIENTS STARTING TNF INHIBITORS WITH MODERATE ACTIVITY THAN WITH HIGH DISEASE ACTIVITY - AN ANALYSIS FROM THE ATTRA REGISTRY}

K. Pavelka ${ }^{1}$, L. Nekvindova ${ }^{2}$, J. Zavada ${ }^{1} .{ }^{1}$ Institute of Rheumatology, Rheumatology, Praha 2, Czech Republic; ${ }^{2}$ Institute of Biostatistics and Analyses LTD, Biotatistics, Brno, Czech Republic

Background: Treat to target guidelines recommend achieving remission or low disease activity in rheumatoid arthritis (RA). In the Czech Republic, anti-TNF therapy has been reimbursed only for patients with highly active RA until 2019, when pts with LDA have been also included in the reimbursement policy.

Objectives: The aim of the study was to compare the results of treatment in patients with rheumatoid arthritis and moderate disease activity (MDA) or high disease activity (HDA) starting the therapy with the first anti-TNF drug in routine clinical practice.

Methods: This was a retrospective cohort study of patients treated with the first anti-TNF drug in the Czech Republic. Propensity score was used to match pts starting anti-TNF in MDA or HDA for other important predictive factors. The propensity score was obtained by logistic regression with 10 covariates (gender, age, disease duration, seropositivity, comedication with MTX, comedication with other csDMARDs, number of previosu csDMARDs, anti-TNF drug),. Within the paired groups, one-dimensional logistic models were then calculated to estimate the odds of remission / low activity after 6 and 12 months of treatment.

Results: A total of 2416 patients were analyzed in the study. 2231 patients had high activity (DAS $28>5.1$ ) and 185 patients had moderate activity (3.2<DAS28 $\leq 5.1$ ) at baseline. After 12 months, the low activity state (DAS28 < 3.2) was achieved by patients with MDA in $74.0 \%$ and patients with HDA in $52.2 \%$, (p $<0.001$ ) (FIG 1). The differences btw groups were significant in all evaluated intervals of $0,3,6,12$ months. Furthermore, a comparison of patients with MDA and HAD by propensity score matching, who no longer differ in the baseline characteristics (except for disease activity and quality of life) was performed. Patients with MDA were $2.4 x$ more likely to achieve remission or low activity according to DAS28-ESR after 6 months and 1.7x more likely to achieve remission or low activity after 12 months of treatment compared to patients with HDA at the beginning of treatment.

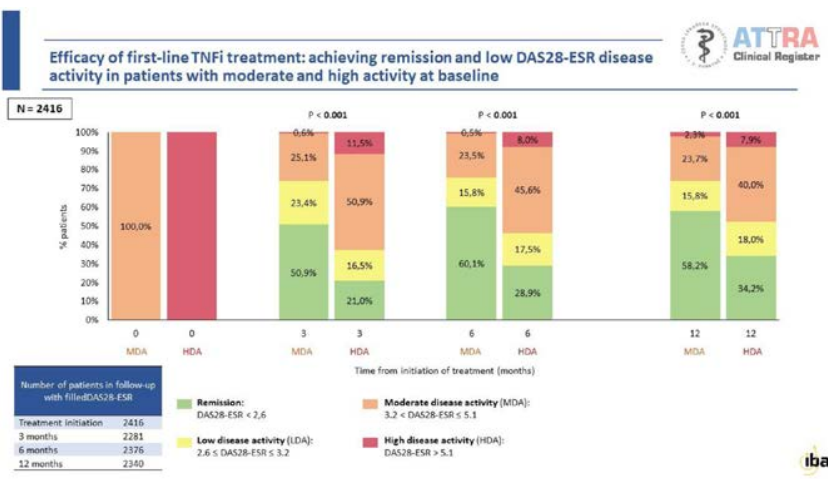

Conclusion: This analysis of the results of treatment with anti-TNF drugs in the ATTRA Registry has shown that starting anti-TNF therapy in patients with RA and MDA leads more often to the target of remission or low activity, than in pts with HDA.

Acknowledgement: Supported by the project of the Ministry of Health Care of the Czech Republic of the conceptual development of the research organization 00023728 Institute of Rheumatology

Disclosure of Interests: Karel Pavelka Speakers bureau: Abbvie, Pfizer, ElliLilly, BMS, UCB, Sanofi, Novartis, Gilead, Paid instructor for: Abbvie, Consultant of: Abbvie, Pfizer, Elli-Lilly, BMS, UCB, Sanofi, Novartis, Gilead, Lucie Nekvindova: None declared, Jakub Zavada Speakers bureau: Abbvie, Elli-Lilly, UCB, Sanofi, Consultant of: Abbvie, UCB, Sanofi, Gilead

DOI: 10.1136/annrheumdis-2021-eular.720

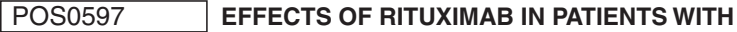 RHEUMATOID ARTHRITIS-RELATED INTERSTITIAL LUNG DISEASE: A SINGLE-CENTRE EXPERIENCE FROM TURKEY}

D. Sahin Eroglu ${ }^{1}$, A. Colaklar ${ }^{2}$, S. Baysal ${ }^{3}$, M. Torgutalp ${ }^{1}$, A. Baygul ${ }^{4}$, M. E. Yayla ${ }^{1}$, T. M. Turgay ${ }^{1}$, G. Kınıkıı ${ }^{1}$, A. Ates ${ }^{1} .{ }^{1}$ Ankara University Faculty of Medicine, Department of Internal Medicine, Division of Rheumatology, Ankara, Turkey; ${ }^{2}$ Ankara University Faculty of Medicine, Department of Radiology, Ankara, Turkey; ${ }^{3}$ Ankara University Faculty of Medicine, Department of Internal Medicine, Ankara, Turkey; ${ }^{4}$ Ankara University Faculty of Medicine, Department of Pulmonology, Ankara, Turkey

Background: Rheumatoid arthritis-related interstitial lung disease (RA-ILD) is the most common type of lung involvement in rheumatoid arthritis (RA). The existence of RA-ILD is associated with worse survival. There is no mainstay treatment for RA-ILD. However, in recent studies, rituximab (RTX) seems to be effective in RA-ILD.

Objectives: To determine the effects of RTX in patients with RA-ILD from a single-centre.

Methods: In our biological treatment outpatient clinic, a retrospective study was conducted in patients with RA who were treated with RTX. Among them, patients with RA-ILD were analysed. For lung response to RTX, progression was defined as a decline of $10 \% \geq$ in forced vital capacity (FVC) and/or a decline of $15 \% \geq$ in diffusion capacity of carbon monoxide (DLCO). Computed tomography of the chest (chest-CT) were integrated to lung response so as to constitute missing data of pulmonary function tests (PFTs).

Results: A total of 165 patients who are followed-up in our biological treatment outpatient clinic have been using RTX for their RA diagnosis. Among 165 patients, 26 (15.8\%) patients with RA-ILD were initiated RTX. Five patients were diagnosed with RA-ILD while using RTX (incidence rate $3 \%$ ). Patients' characteristics were demonstrated in table 1 . Of 26 patients, the most commonly used concomitant disease-modifying antirheumatic drugs was leflunomide (46.2\%) followed by mycophenolate mofetil (11.5), methotrexate (11.5\%) and azathioprine (3.8\%). Twenty-four (92.3\%) patients used steroids. For the evaluation of lung response, 20 patients who had follow-up PFTs and/or chest-CT were compared. 